\title{
Effect of freeze-thaw cycles pretreatment on the vacuum freeze-drying process and physicochemical properties of the dried garlic slices
}

\begin{abstract}
Freeze-thaw cycles (FTC) pretreatment was employed before the vacuum freeze-drying of garlic slices, aimed at improving the drying process and the quality of the end product. Cell viability, water status, internal structure, flavor, chemical composition and thermogravimetric of garlic samples were evaluated. The results indicated that FTC pretreatment reduced the drying time $(22.22 \%-33.33 \%)$ and the energy consumption $(14.25 \%-15.50 \%)$, owing to the water loss, the increase in free water, and the formation of porous structures. The FTC pretreatment improved thermal stability, flavor and chemical composition content of dried products. The antioxidant activity of polysaccharides extracted from FTC pretreated dried products was higher than that of the unpretreated dried product due to the reduction in polysaccharide molecular weight. This research could pave a route for future production of dried garlic slices having good quality by using the FTC pretreatment, with lower energy consumption and shorter drying time.
\end{abstract}

Keyword: Garlic; Freeze-thaw cycles pretreatment; Vacuum freeze-drying process; Physicochemical properties; Polysaccharides 